\title{
Higher phenolic acid excretion in European than Indian volunteers after high polyphenol diet
}

\author{
A. Alkhaldy, C. A. Edwards and E. Combet \\ Human Nutrition, School of Medicine, College of MVLS, University of Glasgow, Glasgow, G3 8SJ, UK
}

The incidence of colorectal cancer (CRC) is high in Scotland and low in India ${ }^{(1)}$. Diet is a major factor. Polyphenol-rich foods have been proposed to have a role in disease prevention and are associated with reduced inflammation and oxidative stress ${ }^{(2)}$. The bioavailability and metabolism of the parent polyphenolics show substantial variability. This may result in the production of different levels of bioactive metabolite in the large intestine where they may influence colorectal cancer ${ }^{(3,4)}$. This variability could be due to i) dietary habits including polyphenol-rich foods, ii) ethnic-specific colonic microflora and iii) differences in absorption of phenolic acids ${ }^{(3,4)}$. The aim of this pilot study was to study how ethnicity impacts on the colonic metabolism of polyphenols.

Healthy volunteers (Indian, recently arrived in UK, $n=8$; European $n=8$, aged 18-45), followed 3-day low and high-polyphenol diets, in a randomised cross-over design with a $>7$ day wash-out. Weighed dietary records and a food frequency questionnaire focusing on polyphenol-rich foods were completed. Urine and faeces were collected at the end of each diet. Metabolites of polyphenols (benzoic acid, phenylacetic acid, mandelic acid, 3-hydroxybenzoic acid, 3-hydroxyphenylacetic acid, 4-hydroxybenzoic acid, 4-hydroxyphenylpropionic acid, vanillic acid, homovanillic acid, 4-hydroxymandelic acid, 3,4-dihydroxybenzoic acid, 3,4-dihydroxyphenylacetic acid, hippuric acid, dihydrocaffeic acid, 4-hydroxy-3-methoxy-phenylpropionic acid, gallic acid, and 4-hydroxyhippuric acid) were measured in urine by GC-MS. Faecal short chain fatty acids (SCFA) were measured by GC-FID. Faecal pH was also measured. Faecal bacteria including Bifidobaceria and Bacteroidetes (modulated by phenolic compounds) and Flavonifractor plautii (a known flavonoid degrader) were measured by real-time quantitative PCR. Results were compared using Wilcoxon sign rank and Mann-Witney $U$ test as appropriate.

As expected, the sum of urinary phenolic acids (PA) was higher after the high-polyphenol diet in both groups (Indian 22.5 (SD 10.2 ) to 71.0 (SD 45.7) $\mu \mathrm{g} / \mathrm{day} ; p=0.01$; European 42.3 (SD 18.3) to 198.3 (SD 65.8); $p=0.0009$ ). Europeans excreted significantly more PA after the high-polyphenol diet $(\Delta 156 \mu \mathrm{g} /$ day, SD 47.6$)$ than Indian volunteers $(\Delta 48.5 \mu \mathrm{g} /$ day, SD 35.5$)(p=0.001)$. Europeans excreted more benzoic acid $(p=0.04)$, 3-hydroxybenzoic acid $(p=0.02), 3$, 4-dihydroxyphenylacetic acid $(p=0.05)$, and hippuric acid $(p=0.001)$. Increasing the intake of polyphenols showed no difference in faecal SCFA within or between the two groups. Mean faecal $\mathrm{pH}$, after the low polyphenol diet, was lower in the Indian group, (6.5 (SD 0.3), than in Europeans (7.2 (SD 0.4); $p=0.003)$. Faecal pH was similar in both groups after the high-polyphenol diet. Faecal concentration of Bifidobacteria was higher $(p=0.04)$ in Indian than European volunteers (10.0 $\log 10$ copies/g wet wt (SD 0.4$)$ versus $9.6 \log 10$ copies/g wet wt (SD 0.3$)$ ). There was no difference between Indian and European volunteers for faecal concentrations of Bacteroidetes or Flavonifractor plautii.

In conclusion, Indian volunteers had less excretion of phenolic acids after the high polyphenol diet than Europeans but there were no differences in specific bacteria known to metabolise polyphenolics.

This work was supported by the Faculty of Applied Medical Sciences - Clinical Nutrition Department, King Abdulaziz University, Jeddah, Kingdom of Saudi Arabia 2010-1013, and Tenovus Scotland.

1. Wild S, Fischbacher C, Brock A et al. (2006) Br J Cancer 7, 1079-1085,

2. Manach C, Williamson G, Morand C et al. (2005) Am J Clin Nutr 81, 230-242.

3. Gross G et al. (2010) J Agric. Food Chem 58(18), 10236-10246.

4. Gardana C, Canzi E, Simenetti P (2009) J Nutr Biochem 20(12), 940-947. 